\title{
Expansion de la liane envahissante Sericostachys scandens Gilg \& Lopr. (Amaranthaceae) en forêt dense humide de montagne: déterminants écologiques et effets sur les plantes herbacées
}

\author{
Richard HABONAYO ${ }^{1,2 *}$, Akomian Fortuné AZIHOU ${ }^{3}$, Gbèwonmèdéa Hospice \\ DASSOU $^{1}$, François HAVYARIMANA ${ }^{4}$, Aristide Cossi ADOMOU ${ }^{1}$ et \\ Bernadette HABONIMANA ${ }^{2}$
}

\author{
${ }^{1}$ Université d'Abomey-Calavi, Faculté des Sciences et Techniques, Laboratoire de Botanique et Ecologie \\ Végétale, 01 BP 4521 Cotonou, République du Bénin. \\ ${ }^{2}$ Université du Burundi, Faculté d'Agronomie et de Bio-Ingénierie, Département des Sciences et Technologies \\ de l'Environnement, BP 2940 Bujumbura-Burundi. \\ ${ }^{3}$ Université d'Abomey-Calavi, Faculté des Sciences Agronomiques, Laboratoire d'Ecologie Appliquée, 01 BP \\ 526 Cotonou, République du Bénin. \\ ${ }^{4}$ Université du Burundi, Faculté des Sciences, Département de Biologie, BP 2700 Bujumbura-Burundi. \\ *Auteur correspondant ; E-mail: habonayorichard@gmail.com; Tél: +22962 941078
}

\section{REMERCIEMENTS}

Les auteurs remercient le Gouvernement du Burundi pour avoir financé ce travail.

\section{RESUME}

Sericostachys scandens, liane envahissante, représente une menace pour la diversité biologique. La présente étude a pour objectif d'évaluer l'effet des trouées, de l'altitude et de la pente sur l'abondance de $S$. scandens dans le Parc National de la Kibira au Burundi; et l'influence de cette liane sur la composition floristique des herbacées. L'abondance de $S$. scandens et des plantes herbacées a été mesurée dans 50 trouées et 50 parcelles témoins sous couverture forestière et soumise à une régression binomiale négative et à une analyse multivariée de la variance sur matrice de dissimilarité. La superficie des trouées a un effet positif sur l'abondance de $S$. scandens $(\mathrm{z}=4,99 ; \mathrm{p}<0,0001)$ alors que l'altitude a un effet négatif $(\mathrm{z}=-12,63 ; \mathrm{p}<0,0001)$. L'invasion des stations forestières par $S$. scandens modifie la composition floristique en herbacées $(p=0,01)$. La stratégie de surveillance du PNK devrait renforcer les patrouilles pour limiter la création de trouées d'origine anthropique. (C) 2019 International Formulae Group. All rights reserved

Mots clés: Trouées, invasion, liane, diversité, Parc, Burundi.

\section{Expansion of the invasive liana Sericostachys scandens Gilg \& Lopr. (Amaranthaceae) in moist mountain forests: ecological determinants and impacts on herbaceous plants}

\begin{abstract}
Sericostachys scandens, an invasive liana, represents a threat to biological diversity. The present study aimed at evaluating of the effect of gaps, altitude and slope on the abundance of $S$. scandens in the Kibira National Park in Burundi; and the influence of this liana on the floristic composition of herbaceous plants. The abundance
\end{abstract}


of $S$. scandens and herbaceous plants was measured in 50 gaps and 50 control plots under forest cover and subjected to negative binomial regression and multivariate variance analysis on dissimilarity matrix. The light gap area has a positive effect on the abundance of $S$. scandens $(\mathrm{z}=4.99, \mathrm{p}<0.0001)$ while the altitude has a negative effect $(\mathrm{z}=-12.63, \mathrm{p}<0,0001)$. The invasion of forest stations by $S$. scandens changes the floristic composition of herbaceous plants $(F=315.026, p=0.01)$. The strategy of KNP surveillance should strengthen patrols to limit the creation of anthropogenic gaps.

(C) 2019 International Formulae Group. All rights reserved

Keywords: Gaps, invasion, liana, diversity, Park, Burundi.

\section{INTRODUCTION}

Les lianes ont été reconnues comme des composantes importantes et intégrales des forêts, en particulier sous les tropiques (van der Heijden et al., 2013; Wyka et al., 2013). Elles sont particulièrement abondantes dans les zones perturbées des forêts naturelles telles que les trouées qu'elles colonisent rapidement (Babweteera et al., 2000; Schnitzer et al., 2000; Ledo et Schnitzer, 2014), notamment par leur propagation végétative (York et al., 2013; Ledo et Schnitzer, 2014).

Les lianes prolifèrent à la faveur de l'exposition à la lumière des trouées (Asner et Martin, 2012) ainsi que la présence de nombreux arbustes qui leur servent de supports (Laurance et al., 2001). La forte colonisation des lianes peut retarder la régénération des arbres dans les trouées pendant plusieurs années. Par exemple, Schnitzer et al. (2000) ont montré qu'une abondance élevée des lianes dans les trouées en forêts naturelles au Panama retarde la régénération des arbres pour plus de 13 ans. L'abondance des lianes peut présenter d'autres conséquences comme l'augmentation de la mortalité des arbres (Schnitzer et al., 2014), la limitation de la croissance et la réduction de la diversité des espèces d'arbres, particulièrement les espèces non pionnières (Schnitzer et Carson, 2010).

Dans les forêts tropicales africaines, la prolifération de Sericostachys scandens Gilg \& Lopr. (Amaranthaceae), une liane envahissante largement distribuée dans les zones montagneuses de l'Afrique centrale et orientale, est inquiétante (Masumbuko, 2011). Cette liane prolifère dans les sites qui ont connu des perturbations humaines, entraîne la mort des arbres et retarde leur régénération. En effet, en se développant, S. scandens grimpe sur les arbres (allant jusqu'à la hauteur de grands arbres), et forme des colonnes compactes entourant les troncs de ces derniers. Arrivée dans la canopée, elle déploie une grande quantité de feuilles qui couvrent toute la plante hôte et ainsi l'étouffe en l'empêchant d'accéder à la lumière. Au fil du temps, les arbres hôtes finissent par sécher et cèdent sous le poids des individus et ramifications enchevêtrées de cette liane (Balezi et al., 2008; Masumbuko, 2011; Habonayo et al., 2019). Lorsque les conditions du milieu ne permettent pas à $S$. scandens de grimper, elle tapisse de grandes surfaces en s'étalant sur la végétation basse et ainsi bloque le développement de la régénération (Liengola, 2008; Masumbuko, 2011). La dominance de $S$. scandens dans les trouées s'accompagne de l'expansion des espèces herbacées comme les fougères (Pteridium sp.) et les espèces du genre Triumfetta (Balezi et al., 2008). Elle est plus répandue dans les forêts de montagnes de la République Démocratique du Congo, du Burundi, du Rwanda et de l'Ouganda (Dhetchuvi et Gakwavu, 2006).

Au Burundi, bien que S. scandens constitue une menace particulière pour la biodiversité du Parc National de la Kibira (PNK) (MEEATU, 2013), le plus grand massif forestier du Burundi, aucune étude concernant sa répartition et ses effets sur le développement d'autres espèces n'y a été faite. L'identification des facteurs susceptibles de rendre le PNK vulnérable à $S$. scandens est une voie pour contrôler et réduire ses effets sur cet écosystème. 
L'objectif général de la présente étude est d'identifier les déterminants écologiques de l'abondance de $S$. scandens dans le PNK et l'influence de cette liane sur la composition floristique des herbacées. Les objectifs spécifiques fixés sont de (1) comparer la densité de $S$. scandens entre les trouées et les parcelles sous canopée intacte, (2) analyser l'effet de la superficie des trouées, de l'altitude et de la pente sur l'abondance de $S$. scandens, et (3) analyser l'effet de l'abondance de $S$. scandens sur la diversité taxonomique et la composition floristique des herbacées.

\section{MATERIEL ET METHODES Milieu d'étude}

Le PNK est situé au nord-ouest du Burundi sur la crête Congo-Nil (Figure 1). D'une superficie de 40.000 ha, une longueur de plus ou moins $80 \mathrm{~km}$ et une largeur moyenne de $8 \mathrm{~km}$, il constitue le principal massif forestier du pays (Krug, 1993). Il s'étend de Bugarama au sud jusqu'à la frontière rwandaise au nord où il se prolonge par la forêt de Nyungwe au Rwanda. Il se localise entre $2^{\circ} 36^{\prime} 52^{\prime \prime}$ et $3^{\circ} 17^{\prime} 08^{\prime \prime}$ de latitude sud et $29^{\circ} 13^{\prime} 31^{\prime \prime}$ et $29^{\circ} 39^{\prime} 09^{\prime \prime}$ de longitude est. Son altitude varie entre 1600 et $2666 \mathrm{~m}$ (Krug, 1993). Il s'étend sur quatre provinces, à savoir Bubanza et Cibitoke à l'ouest d'une part et Muramvya et Kayanza à l'est d'autre part, couvrant les communes Musigati, Rugazi, Bukinanyana, Mabayi, Bukeye, Muramvya, Kabarore, Matongo et Muruta. Selon Gourlet (1986), le PNK est subdivisé en quatre blocs ou secteurs: Teza (5794 ha), Rwegura (12424 ha), Mabayi (6359 ha) et Musigati (15424 ha).

Le relief du PNK est marqué par de fortes pentes de part et d'autre de la Crête Congo-Nil, davantage marquées sur le versant occidental (INECN, 1992). La ligne de crête délimite deux bassins versants communément appelés «Bassin du Nil» à l'est et «Bassin du Congo» à l'ouest.

Le climat du PNK est de type tropical d'altitude à tendance tempérée marqué par son caractère montagnard. Les températures moyennes sont les plus basses du pays, variant entre 14 et $20^{\circ} \mathrm{C}$ selon l'altitude (Krug, 1993). La température moyenne mensuelle enregistrée à la station météorologique de Rwegura au cours des années 1986 à 2015 est de $16,4{ }^{\circ} \mathrm{C}$ (IGEBU, 2015). Les précipitations annuelles sont supérieures à $1800 \mathrm{~mm}$ et atteignent parfois $2000 \mathrm{~mm}$. L'humidité se situe entre 60 et $90 \%$ pendant toute l'année mais reste généralement proche de la moyenne évaluée à $75 \%$. Les brouillards sont fréquents en altitude, surtout dans les vallées (IGEBU, 2001).

A l'ouest du PNK, les sols sont argileux et faiblement ferralitiques. Sur le versant Est, deux zones de sols sont identifiées: les lithosols (Nord de Teza) et les andosols (dans la région de Teza) (Cazenave-Piarrot et al., 1979).

La végétation du PNK est caractérisée par la forêt ombrophile de montagne. En fonction des critères écologiques, floristiques et phytogéographiques, Lewalle (1972) a distingué trois étages dans cette végétation montagnarde: (i) étage inférieur compris entre 1600 et $1900 \mathrm{~m}$ d'altitude caractérisé par une forte densité et l'intrication des strates arborescentes dont les arbres peuvent atteindre $25 \mathrm{~m}$ de hauteur; (ii) étage moyen allant de 1900 à $2250 \mathrm{~m}$ d'altitude correspondant à une forêt constituée d'arbres atteignant $30 \mathrm{~m}$ et parfois $40 \mathrm{~m}$ de hauteur et (iii) étage supérieur (2250 m à $2450 \mathrm{~m}$ ) où se développe une forêt composée d'arbres atteignant $15 \mathrm{~m}$ de hauteur sauf quelques individus exceptionnels pouvant atteindre $25 \mathrm{~m}$ de hauteur.

\section{Collecte des données}

La collecte des données a été réalisée dans les quatre secteurs du PNK en considérant les deux bassins versants (bassin du Nil et bassin du Congo) et les trois niveaux d'altitude (de 1600 à $1900 \mathrm{~m}$, de 1900 à $2250 \mathrm{~m}$ et de 2250 à $2450 \mathrm{~m}$ ) correspondant aux trois étages de végétation du PNK. Les observations de terrain ont permis de noter l'absence de forêts au niveau d'altitude 1600-1900 m du côté bassin versant du Nil. Par conséquent, les données ont été collectées dans cinq niveaux d'altitude sur l'ensemble du parc. Par niveau d'altitude, les observations ont été faites dans 
20 sites (10 trouées et 10 parcelles situées entièrement dans une zone sous canopée intacte), soit au total 100 sites équitablement répartis entre les trouées et les parcelles sous canopée intacte (parcelles témoins) à l'échelle du parc. Pour chaque site, les coordonnées géographiques (altitude, latitude et longitude prises au centre du site) et la pente (mesurée en considérant le sens où elle est la plus grande) ont été déterminées au moyen d'un GPS Garmin 76 et un clinomètre Suunto respectivement.

L'échantillonnage a été précédé par la détermination des dimensions de chaque trouée à savoir l'axe principal ou plus grande longueur (L) et un certain nombre de segments de ligne $\left(1_{1}, 1_{2}, 1_{3}, 1_{4}\right.$, etc. $)$ partant d'une bordure à l'autre de la trouée et disposés de manière à ce qu'ils coupent orthogonalement l'axe principal en des endroits équidistants entre eux (Figure 2). Ces données ont permis de calculer la surface (S) de la trouée par la formule:

$\mathrm{S}=\mathrm{I}(\mathrm{A}+\mathrm{B}+\mathrm{C}+\mathrm{D}+\ldots+\mathrm{N})$ où $\mathrm{I}$ est l'équidistance entre les petits segments de ligne $\left(1_{1}, l_{2}, l_{3}, 1_{4}, \ldots, l_{n}\right)$ au niveau de l'axe principal et $\mathrm{A}, \mathrm{B}, \mathrm{C}, \mathrm{D}, \ldots, \mathrm{N}$ sont leurs longueurs respectives. $\mathrm{La}$ parcelle témoin a été déterminée en reprenant la même forme et les mêmes dimensions que celles de la trouée qui lui est appariée (Grainger et Rudi, 2012). Les deux sites étaient séparés par une distance variant entre 100 et $200 \mathrm{~m}$ et ont été parcourus dans une direction sud-nord. Ils ont été choisis de façon à ce que qu'ils soient situés dans des conditions topographiques similaires et à une même altitude.

Dans chaque trouée et parcelle témoin, tous les individus de $S$. scandens ayant un diamètre supérieur ou égal à $1 \mathrm{~cm}$ à $130 \mathrm{~cm}$ du point d'enracinement ont été inventoriés à l'aide d'un mètre ruban, suivant le protocole standard proposé par Gerwing et al. (2006). En plus de l'échantillonnage de $S$. scandens, d'autres espèces ont également été inventoriées. Etant donné que certaines trouées étaient d'une surface inférieure à $400 \mathrm{~m}^{2}$ qui est la taille minimale pour l'échantillonnage des espèces de la strate arborescente (Lewalle,
1972), cet inventaire n'a concerné que les espèces herbacées, mettant ainsi de côté les espèces arborées quel que soit leur statut structural.

D'après Senterre (2005), en forêts denses d'Afrique centrale, une surface de l'ordre de $200 \mathrm{~m}^{2}$ permet l'observation de toutes les espèces herbacées d'une station donnée. Dans chaque trouée/parcelle témoin, l'échantillonnage a été effectué dans deux placettes carrées de $100 \mathrm{~m}^{2}$ de superficie chacune. Pour une estimation plus aisée du recouvrement des espèces, les placettes ont été découpées en quatre carrés de $5 \mathrm{~m}$ x $5 \mathrm{~m}$.

Dans chaque carré, chaque espèce rencontrée a été affectée d'un indice ou coefficient d'abondance-dominance selon l'échelle de Braun-Blanquet (1964) présentée par Meddour (2011) (Tableau 1) et sa valeur moyenne du coefficient de recouvrement $(\mathrm{CR} \%)$ a été calculée. En effet, on transforme chaque abondance-dominance en pourcentage de recouvrement moyen à l'aide de l'échelle indiquée, puis on additionne, pour cette espèce, les valeurs correspondantes; on divise le total obtenu par le nombre de relevés et on multiplie par cent (De Foucault, 1980 cité par Meddour, 2011):

$$
\mathrm{CR} \%=\frac{\sum \text { des recouvrements moyens x } 100}{\text { nombre de relevés }}
$$

Où recouvrement moyen représente la valeur obtenue après transformation de chaque abondance-dominance et nombre de relevés représente le nombre de carrés de $5 \mathrm{~m}$ x $5 \mathrm{~m}$ de chaque trouée/parcelle témoin où une espèce donnée a été inventoriée.

Pendant l'échantillonnage, les spécimens en tiges avec feuilles, fleurs et/ou en fruits des différentes espèces ont été récoltés et mis en herbier. La détermination des noms scientifiques des espèces a été réalisée grâce aux divers ouvrages notamment ceux de Reekmans et Niyongere (1983) et Habiyaremye et Nzigidahera (2016). L'Herbarium de l'Université du Burundi et celui de l'Office Burundais pour la Protection de l'Environnement (OBPE) ont été également consultés ainsi que divers spécialistes locaux. 
Les noms scientifiques ainsi que les familles des différentes espèces de plantes ont été validés après la vérification des noms actualisés dans la base de données des plantes africaines sur le site http://www.villege.ch/musinfo/bd/cjb/africa/recherche.php.

\section{Traitement des données}

Pour chaque trouée/parcelle, la densité de $S$. scandens ( $d$; individus/ha) a été calculée par la formule suivante (Masumbuko, 2011):

$$
d=\frac{\mathrm{N}}{\mathrm{S}}
$$

Où $\mathrm{N}$ est le nombre d'individus et $\mathrm{S}$ est la surface ramenée à l'hectare.

Le test de Wilcoxon à deux échantillons appariés a été exécuté pour comparer la densité (nombre d'individus par hectare) de $S$. scandens entre les trouées et les parcelles témoins. Avant d'appliquer le test de Wilcoxon, la normalité des données a été vérifiée à l'aide du test de normalité de Shapiro-Wilk. Une régression multiple sur les données de comptage a été utilisée pour tester l'effet de la superficie de la trouée, de l'altitude et de la pente sur la densité de $S$. scandens au niveau des trouées. Après vérification de la surdispersion des données, la régression binomiale négative a été identifiée comme étant la plus appropriée. La corrélation entre les variables indépendantes (altitude, superficie, pente) prises deux a deux a été testée afin d'éviter l'effet de colinéarité dans les modèles initiaux. La régression de quasi-Poisson a été exécutée pour tester l'effet de la densité de $S$. scandens sur la richesse spécifique, la diversité en genres et la diversité en familles des espèces herbacées. Une analyse multivariée de la variance sur matrice de dissimilarité avec 99 permutations (ADONIS) a été exécutée pour tester la variation de la composition floristique des espèces herbacées en fonction de l'unité d'échantillonnage (trouées versus placettes témoins), de la superficie et de l'altitude. La valeur indicatrice a été calculée pour identifier les espèces herbacées caractéristiques des trouées et de la végétation sous couvert intact (Dufrêne et Legendre, 1997). Toutes les analyses ont été faites au moyen du logiciel $\mathrm{R}$ version 3.4.2 ( $\mathrm{R}$ Core Team, 2017). La régression binomiale négative a été exécutée avec le package 'MASS' (Venable et Ripley, 2002) et le coefficient de détermination de Nagelkerke calculée avec le package 'fmsb' (Nakazawa, 2017). ADONIS a été réalisé sous le package 'vegan' (Oksanen et al., 2017) et les valeurs indicatrices calculées avec le package 'labdsv' (Roberts, 2016). Les résultats ont été jugés statistiquement significatifs avec une probabilité inférieure à $5 \%$.

Tableau 1: Coefficients d'abondance-dominance selon Braun-Blanquet (1964).

\begin{tabular}{lcc}
\hline Recouvrement moyen & Abondance-dominance & Coefficient de pondération \\
\hline $0-1 \%$ & + & 0,5 \\
$1-5 \%$ & 1 & 3 \\
$5-25 \%$ & 2 & 15 \\
$25-50 \%$ & 3 & 37,5 \\
$50-75 \%$ & 4 & 62,5 \\
$75-100 \%$ & 5 & 87,5 \\
\hline
\end{tabular}




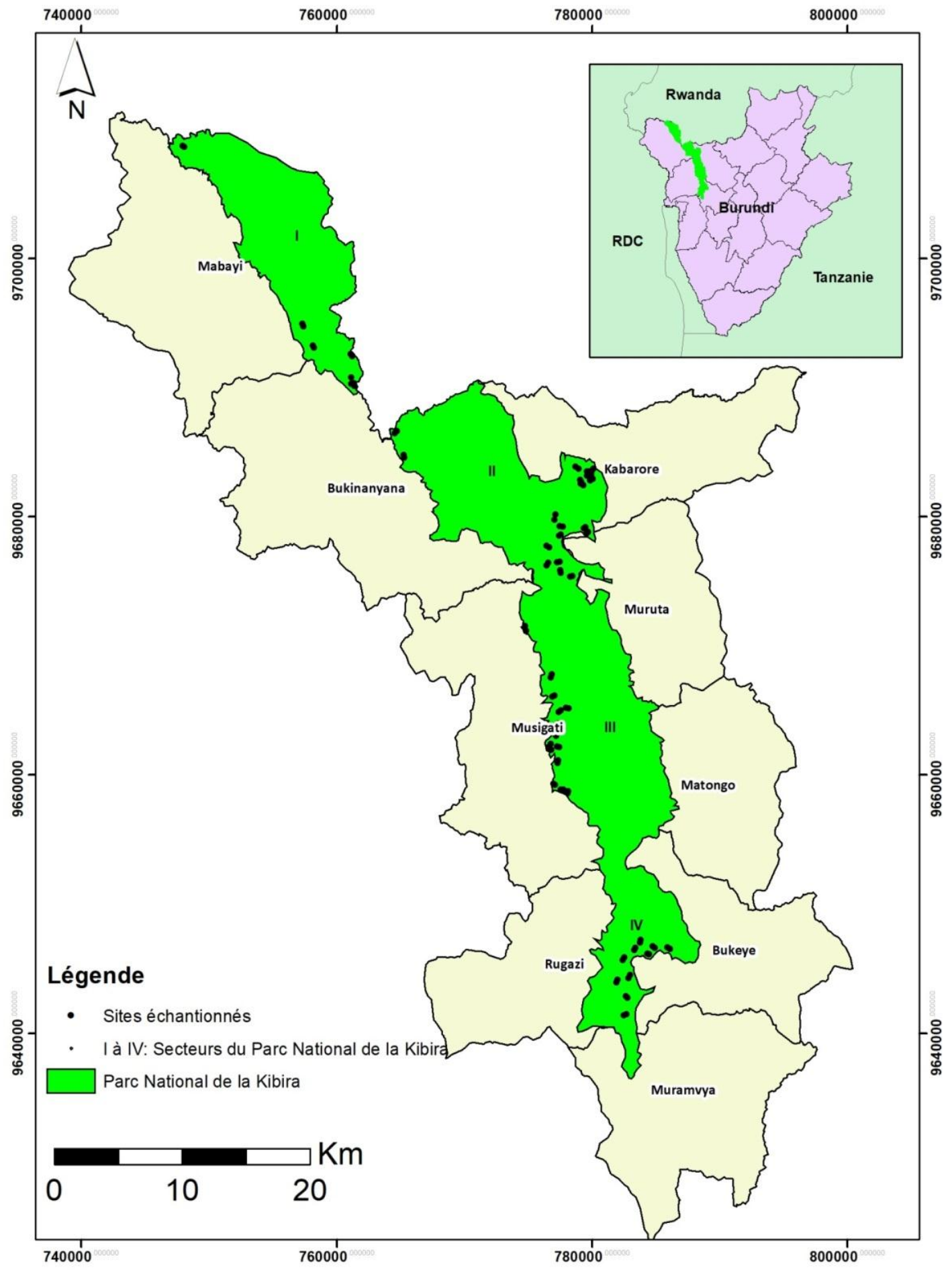

Figure 1: Localisation du Parc National de la Kibira (PNK): (A) localisation du PNK par rapport au Burundi, (B) localisation des sites d'échantillonnage dans les secteurs du PNK ainsi que la localisation de ces derniers par rapport aux communes administratives du Burundi couvrant ce parc $(I=$ secteur Mabayi, II = secteur Rwegura, III = secteur Musigati, IV = secteur Teza). 


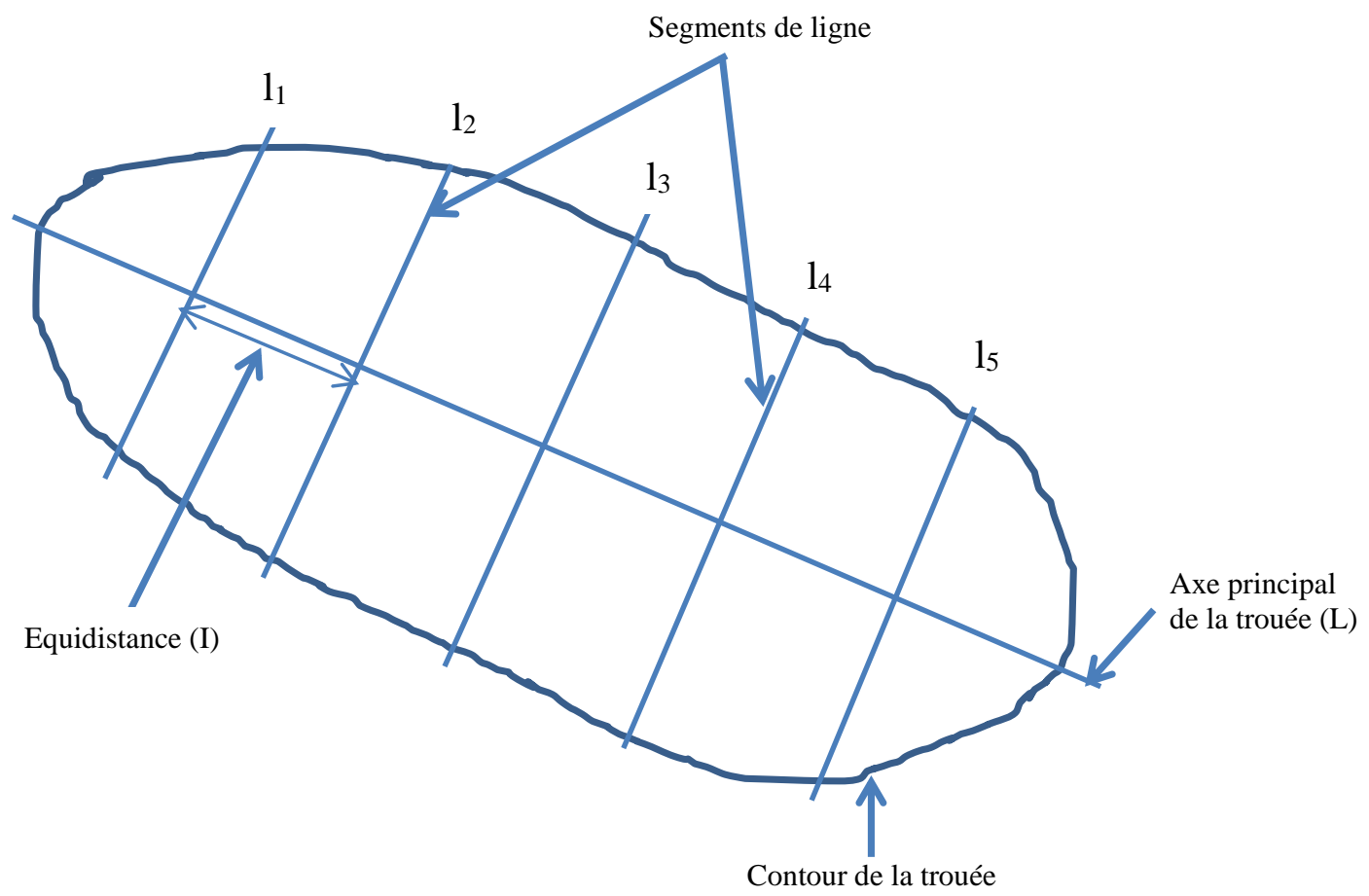

Figure 2: Détermination des dimensions d'une trouée. L'axe principal ou plus grande longueur (L) et un certain nombre de segments de ligne $\left(1_{1}, 1_{2}, 1_{3}, 1_{4}\right.$, etc.) partant d'une bordure à l'autre de la trouée et disposés de manière à ce qu'ils coupent orthogonalement l'axe principal en des endroits équidistants entre eux ont été définis et mesurés. Ces données ont permis de calculer la surface $(\mathrm{S})$ de la trouée par la formule: $\mathrm{S}=\mathrm{I}(\mathrm{A}+\mathrm{B}+\mathrm{C}+\mathrm{D}+\ldots+\mathrm{N})$ où I est l'équidistance entre les petits segments de ligne $\left(1_{1}, 1_{2}, 1_{3}, 1_{4}, \ldots, 1_{n}\right)$ au niveau de l'axe principal et $A, B, C, D, \ldots, N$ sont leurs longueurs respectives.

\section{RESULTATS}

\section{Variation de la densité de Sericostachys scandens entre les trouées et les parcelles témoins}

La densité (nombre d'individus par ha) moyenne de $S$. scandens s'élève à $4830 \pm 190$ et à $19 \pm 6,31$ respectivement dans les trouées et les parcelles témoins. Les résultats du test de Wilcoxon à deux échantillons appariés ont montré qu'il y a une différence hautement significative des densités de $S$. scandens entre les trouées et les parcelles témoins (test de Wilcoxon, $\mathrm{W}=1275 ; \mathrm{p}<0,0001)$. Ainsi, la liane envahissante $S$. scandens est 254 fois plus abondante dans les trouées comparativement à la végétation sous couverture fermée.

\section{Déterminants écologiques de l'abondance de Sericostachys scandens dans les trouées}

Les modèles exécutés ont permis d'identifier l'altitude, la superficie de la trouée et la pente comme variables influençant significativement l'abondance de S. scandens (Tableau 2).

Le meilleur modèle de régression (modèle $1 ; \mathrm{R}^{2}=0,987 ; \mathrm{AIC}=470,03$ ) met en évidence l'effet négatif de l'altitude sur la densité de la liane contre un effet positif de la superficie de la trouée (Tableau 2). Ainsi, la liane envahit plus facilement les zones de faible altitude et les zones de grandes trouées. Le modèle 2 renseigne également sur un effet positif de la pente sur l'abondance de la liane.

\section{Effet de l'expansion de Sericostachys scandens sur la diversité floristique des} herbacées

Un total de 78 espèces végétales réparties dans 74 genres et 44 familles ont été inventoriées. Les résultats de la régression de quasi-Poisson (Tableau 3) indiquent que la densité de $S$. scandens influence 
significativement la diversité des herbacées du PNK. Ainsi, elle a un effet négatif sur la richesse spécifique $\left(R^{2}=0,59 ; z=-6,711 ; p<\right.$ $0,0001)$, la diversité en genres $\left(R^{2}=0,62 ; z=-\right.$ $6,950 ; \mathrm{p}<0,0001)$ et la diversité en familles $\left(R^{2}=0,50 ; z=-5,448 ; p<0,0001\right)$. En d'autres termes, l'invasion par $S$. scandens affecte négativement la diversité taxonomique dans la formation étudiée.

Les résultats de l'ADONIS (Tableau 4) ont indiqué une variation significative de la composition floristique des herbacées entre les trouées envahies par la liane et les parcelles témoins $(\mathrm{p}=0,01)$. L'unité d'échantillonnage (trouée versus parcelle témoin) explique à elle seule $76 \%$ de la variation de la composition floristique des herbacées entre les trouées et les parcelles témoins. Par contre, ni la superficie de la trouée, ni l'altitude n'a un effet significatif sur la composition floristique des herbacées $(\mathrm{p}=0,37$ et $\mathrm{p}=0,18$ respectivement). Aucune interaction entre les facteurs n'a été significative.

Les résultats du calcul de la valeur indicatrice ont mis en exergue 20 espèces indicatrices des trouées envahies par $S$. scandens (Tableau 5) et 43 espèces indicatrices des parcelles témoins à couverture forestière fermée (Tableau 6). Ainsi, Pteridium aquilinum (L.) Kuhn a été recensée dans toutes les trouées envahies. A l'opposé, Brillantaisia cicatricosa Lindau et Chassalia subochreata (De Wild.) Robyns l'ont été dans toutes les parcelles témoins. Les autres espèces les plus indicatrices (valeur indicatrice $\geq 0,80$ ) sont Gynura scandens O.Hoffm, Urera hypselodendron (Hochst. ex A.Rich.) Wedd., Clerodendrum johnstonii Oliver, Ageratum conyzoides L., Rubus apetalus Poir., Crassocephalum montuosum (S. Moore) Milne-Redh., Triumfetta tomentosa Bojer et Ipomoea involucrata $\mathrm{P}$. Beauv. pour les trouées (Tableau 5) d'une part et Asplenium elliottii C.H.Wright, Calanthe sylvatica (Thouars.) Lindl., Pilea rivularis Wedd., Elatostema monticola Hook. f., Galiniera coffeoides Delile, Impatiens burtonii Hook.f., Notholaena inaequalis Kunze, Rutidea orientalis Bridson, Piper capense L.f., Cordia sp., Achyrospermum micranthum Perkins et Sanicula elata Buch.-Ham. ex. D. Don pour les parcelles sous couvert fermé (Tableau 6) d'autre part.

Tableau 2: Résultats de la régression de Négative binomiale montrant l'effet de la superficie, de l'altitude et de la pente sur la densité de $S$. scandens dans les trouées du Parc National de la Kibira.

\begin{tabular}{llllllll}
\hline & Estimate & Std.Error & z value & $\mathbf{P}(>|\mathbf{z}|)$ & $\mathbf{R}^{2}$ & AIC \\
\hline Modèle 1 & Constante & 6,94 & 0,128 & 54,18 & $<0,0001$ & 0,987 & 470,03 \\
& Altitude & $-0,001$ & 0,000 & $-12,63$ & $<0,0001$ & & \\
& Superficie & 0,000 & 0,000 & 4,99 & $<0,0001$ & & \\
\hline Modèle 2 & Constante & 5,225 & 0,092 & 56,76 & $<0,0001$ & 0,676 & 518,16 \\
& Pente & 0,005 & 0,001 & 5,26 & $<0,0001$ & & \\
& Superficie & 0,000 & 0,000 & 4,13 & $<0,0001$ & & \\
\cline { 3 - 6 } & & & & & &
\end{tabular}


Tableau 3: Résultats de la régression de quasi-Poisson montrant l'effet de la densité de $S$. scandens sur la richesse spécifique, la diversité en genres et la diversité en familles des espèces herbacées du Parc National de la Kibira.

\begin{tabular}{lllllll}
\hline Variable expliquée & & Estimate & Std.Error & $\mathbf{z}$ value & $\mathbf{P}(>|\mathbf{z}|)$ & $\mathbf{R}^{2}$ \\
\hline Richesse spécifique & & & & & & \\
& Constante & 3,200 & 0,092 & 34,699 & $<0,0001$ & 0,587 \\
& Densité & $-0,000$ & 0,000 & $-6,711$ & $<0,0001$ & \\
\hline Diversité en genres & & & & & & \\
& Constante & 3,270 & 0,104 & 31,450 & $<0,0001$ & 0,624 \\
& Densité & $-0,000$ & 0,000 & $-6,950$ & $<0,0001$ & \\
\hline Diversité en familles & & & & & & \\
& Constante & 2,830 & 0,134 & 21,148 & $<0,0001$ & 0,497 \\
& Densité & $-0,000$ & 0,000 & $-5,448$ & $<0,0001$ & \\
\hline P= Probabilité, $\mathrm{R}^{2}=$ coefficient de détermination & & & & &
\end{tabular}

Tableau 4: Résultats de analyse multivariée de la variance sur matrice de dissimilarité (ADONIS) montrant la variation de la composition floristique des herbacées entre les trouées envahies par $S$. scandens et les parcelles témoins dans le Parc National de la Kibira.

\begin{tabular}{|c|c|c|c|c|c|c|}
\hline Source de variation & Df & Sums of Sqs & Mean Sqs & F.Model & $\mathbf{R}^{2}$ & $\mathbf{P}(>\mathbf{F})$ \\
\hline Superficie & 1 & 0,069 & 0,069 & 1,018 & 0,002 & 0,37 \\
\hline Unité & 1 & 21,415 & 21,415 & 315,026 & 0,760 & 0,01 \\
\hline Altitude & 2 & 0,222 & 0,111 & 1,634 & 0,008 & 0,18 \\
\hline Superficie x Unité & 1 & 0,061 & 0,061 & 0,894 & 0,002 & 0,39 \\
\hline Superficie x Altitude & 2 & 0,102 & 0,051 & 0,747 & 0,004 & 0,52 \\
\hline Unité x Altitude & 2 & 0,203 & 0,102 & 1,494 & 0,007 & 0,15 \\
\hline Superficie x Unité x Altitude & 2 & 0,109 & 0,055 & 0,804 & 0,004 & 0,47 \\
\hline Residuals & 88 & 5,982 & 0,068 & 0,2124 & & \\
\hline Total & 99 & 28,164 & 1 & & & \\
\hline
\end{tabular}


Tableau 5: Valeurs indicatrices des espèces herbacées des trouées envahies par $S$. scandens dans le Parc National de la Kibira.

\begin{tabular}{|c|c|c|c|c|}
\hline Familles & Espèces végétales & VI & $\mathbf{P}$ & Stations forestières \\
\hline Dennstaedtiaceae & Pteridium aquilinum (L.) Kuhn & 1,000 & 0,001 & $\begin{array}{l}\text { milieu ouvert (jachères) } \\
\text { milieu ouvert (friches }\end{array}$ \\
\hline Asteraceae & Gynura scandens O.Hoffm. & 0,959 & 0,001 & herbacées) \\
\hline Urticaceae & $\begin{array}{l}\text { Urera hypselodendron (Hochst. ex } \\
\text { A.Rich.) Wedd. }\end{array}$ & 0,957 & 0,001 & $\begin{array}{l}\text { milieu ouvert (friches } \\
\text { herbacées) } \\
\text { milieu ouvert (friches }\end{array}$ \\
\hline Verbenaceae & Clerodendrum johnstonii Oliver. & 0,955 & 0,001 & herbacées) \\
\hline Asteraceae & Ageratum conyzoides $\mathrm{L}$. & 0,899 & 0,001 & milieu ouvert (jachères) \\
\hline Rosaceae & Rubus apetalus Poir. & 0,892 & 0,001 & milieu ouvert (jachères) \\
\hline Asteraceae & $\begin{array}{l}\text { Crassocephalum montuosum (S. Moore) } \\
\text { Milne-Redh. }\end{array}$ & 0,879 & 0,001 & milieu ouvert (jachères) \\
\hline Tiliaceae & Triumfetta tomentosa Bojer & 0,875 & 0,001 & $\begin{array}{l}\text { milieu ouvert (friches } \\
\text { herbacées) } \\
\text { milieu ouvert (friches }\end{array}$ \\
\hline Convolvulaceae & Ipomoea involucrata $\mathrm{P}$. Beauv. & 0,812 & 0,001 & herbacées) \\
\hline Asteraceae & Galinsoga sp. & 0,737 & 0,001 & milieu ouvert (jachères) \\
\hline Asteraceae & Conyza sumatrensis (Retz.) E.Walker & 0,639 & 0,001 & milieu ouvert (jachères) \\
\hline Asteraceae & Vernonia sp. & 0,540 & 0,001 & milieu ouvert (jachères) \\
\hline Asteraceae & Vernonia kirungae R. E. Fr. & 0,519 & 0,001 & milieu ouvert (jachères) \\
\hline Asteraceae & $\begin{array}{l}\text { Anisopappus africanus (Hook.f.) Oliv. } \\
\& \text { Hiern }\end{array}$ & 0,498 & 0,001 & milieu ouvert (jachères) \\
\hline Asteraceae & Helichrysum panduratum O.Hoffm. & 0,451 & 0,001 & milieu ouvert (jachères) \\
\hline Asteraceae & Vernonia auriculifera Hiern & 0,419 & 0,001 & milieu ouvert (jachères) \\
\hline Asteraceae & Microglossa pyrifolia (Lam.) Kuntze & 0,415 & 0,001 & milieu ouvert (jachères) \\
\hline Rubiaceae & Virectaria major (K. Schum.) Verdc. & 0,329 & 0,001 & milieu ouvert (jachères) \\
\hline Rhamnaceae & Gouania longispicata Engl. & 0,293 & 0,002 & milieu ouvert (jachères) \\
\hline Lobeliaceae & Lobelia giberroa Hemsl. & 0,269 & 0,001 & milieu ouvert (jachères) \\
\hline
\end{tabular}

$\mathrm{VI}=$ Valeur indicatrice, $\mathrm{P}=$ Probabilité.

Tableau 6: Valeurs indicatrices des espèces herbacées des parcelles témoins dans le Parc National de la Kibira.

\begin{tabular}{lllll}
\hline Familles & Espèces végétales & VI & P & Stations forestières \\
\hline Acanhtaceae & Brillantaisia cicatricosa Lindau & 1,000 & 0,001 & forêt secondaire mature \\
Rubiaceae & Chassalia subochreata (De Wild.) Robyns & 1,000 & 0,001 & forêt secondaire mature \\
Aspleniaceae & Asplenium elliottii C.H.Wright & 0,980 & 0,001 & forêt primaire \\
Orchidaceae & Calanthe sylvatica (Thouars.) Lindl. & 0,980 & 0,001 & forêt primaire \\
Urticaceae & Pilea rivularis Wedd. & 0,979 & 0,001 & forêt secondaire mature \\
Urticaceae & Elatostema monticola Hook. f. & 0,960 & 0,001 & forêt secondaire mature \\
Rubiaceae & Galiniera coffeoides Delile & 0,960 & 0,001 & forêt secondaire mature
\end{tabular}




\begin{tabular}{|c|c|c|c|c|}
\hline Familles & Espèces végétales & VI & $\mathbf{P}$ & Stations forestières \\
\hline Balsaminaceae & Impatiens burtonii Hook.f. & 0,939 & 0,001 & forêt primaire \\
\hline Pteridaceae & Notholaena inaequalis Kunze & 0,900 & 0,001 & forêt primaire \\
\hline Rubiaceae & Rutidea orientalis Bridson & 0,900 & 0,001 & forêt secondaire mature \\
\hline Piperaceae & Piper capense L.f & 0,899 & 0,001 & forêt primaire \\
\hline Boraginaceae & Cordia sp. & 0,820 & 0,001 & - \\
\hline Lamiaceae & Achyrospermum micranthum Perkins & 0,800 & 0,001 & forêt primaire \\
\hline Apiaceae & Sanicula elata Buch.-Ham. ex. D. Don & 0,800 & 0,001 & forêt secondaire mature \\
\hline Dracaenaceae & Dracaena afromontana Mildbr. & 0,718 & 0,001 & forêt secondaire mature \\
\hline Rubiaceae & $\begin{array}{l}\text { Rytigynia kivuensis (K. Krause) Robyns. } \\
\text { Clausena anisata (Willd.) Hook. f. ex }\end{array}$ & 0,560 & 0,001 & forêt primaire \\
\hline Rutaceae & Benth. & 0,380 & 0,001 & - \\
\hline Euphorbiaceae & Erythrococca bongensis $\mathrm{Pax}$ & 0,380 & 0,001 & forêt primaire \\
\hline Myrsinaceae & Embelia schimperi Vatke & 0,360 & 0,001 & - \\
\hline Apocynaceae & Tacazzea apiculata Oliv. & 0,356 & 0,001 & forêt secondaire mature \\
\hline Sapindaceae & Allophylus sp. & 0,340 & 0,001 & - \\
\hline Menispermaceae & $\begin{array}{l}\text { Stephania abyssinica (Quart.-Dill. \& A. } \\
\text { Rich.) Walp. }\end{array}$ & 0,340 & 0,001 & - \\
\hline Aquifoliaceae & Ilex mitis (L.) Radlk. & 0,320 & 0,001 & - \\
\hline Celastraceae & $\begin{array}{l}\text { Maytenus arbutifolia (Hochst. ex A. Rich.) } \\
\text { R. Wilczek }\end{array}$ & 0,320 & 0,001 & - \\
\hline Euphorbiaceae & Acalypha ornata Hochst-ex A. Rich. & 0,300 & 0,001 & - \\
\hline Flacourtiaceae & Dasylepis racemosa Oliv. & 0,300 & 0,001 & - \\
\hline Amaranthaceae & Celosia sp. & 0,283 & 0,001 & - \\
\hline Cyatheaceae & Alsophila dregei (Kunze) R.M. Tryon & 0,280 & 0,001 & - \\
\hline Poaceae & Arundinaria alpina K. Schum. & 0,280 & 0,006 & forêt primaire \\
\hline Anthericaceae & Chlorophytum sparsiflorum Baker & 0,280 & 0,001 & - \\
\hline Apiaceae & Sanicula elata Buch.-Ham. ex. D. Don & 0,260 & 0,001 & forêt secondaire mature \\
\hline Musaceae & Ensete ventricosum (Welw.) Cheesman & 0,220 & 0,002 & forêt secondaire mature \\
\hline Cucurbitaceae & Lagenaria rufa (Gilg) C. Jeffrey & 0,220 & 0,001 & - \\
\hline Hypericaceae & Hypericum revolutum Vahl & 0,200 & 0,001 & - \\
\hline Flacourtiaceae & Lindackeria kivuensis P. Bamps & 0,200 & 0,001 & - \\
\hline Fabaceae & Desmodium repandum (Vahl) DC. & 0,168 & 0,011 & forêt secondaire mature \\
\hline Dracaenaceae & Dracaena laxissima Engl. & 0,160 & 0,003 & forêt secondaire mature \\
\hline Pteridaceae & Adiantum thalictroides Willd. ex Schltdl. & 0,140 & 0,017 & - \\
\hline Fabaceae & Amphicarpa africana (Hook. F.) Harms & 0,140 & 0,015 & - \\
\hline Begoniaceae & Begonia meyeri-johannis Engl. & 0,140 & 0,01 & - \\
\hline Cucurbitaceae & $\begin{array}{l}\text { Raphidiocystis phyllocalyx C. Jeffrey \& } \\
\text { Keraudren }\end{array}$ & 0,140 & 0,014 & forêt secondaire mature \\
\hline Poaceae & Setaria plicatilis (Hochst.) Hack. ex Engl. & 0,140 & 0,012 & - \\
\hline Solanaceae & Solanum sp. & 0,120 & 0,03 & - \\
\hline
\end{tabular}




\section{DISCUSSION}

\section{Variation de la densité de Sericostachys scandens entre les trouées et les stations sous canopée fermée}

Les résultats ont mis en relief que la liane est significativement plus abondante dans les trouées qu'en milieu sous canopée intacte où elle est presque absente. Plusieurs chercheurs dont Balezi et al. (2008), Masumbuko (2011) et Bongers et Ewango (2015) ont également rapporté que cette liane se répand rapidement dans les trouées. En effet, les trouées sont caractérisées par une forte lumière (Asner et Martin, 2012) faisant partie des conditions cruciales au développement des lianes qui sont des plantes héliophiles (Ledo et Schnitzer 2014; Schnitzer et al., 2015).

\section{Déterminants de la densité de Sericostachys scandens dans les trouées}

Les résultats ont montré que les facteurs essentiels de variation de l'abondance de $S$. scandens au niveau des trouées sont l'altitude dont l'effet est négatif et la superficie de la trouée dont l'effet est positif. L'effet négatif de l'altitude sur l'abondance de $S$. scandens a été également mis en relief par Masumbuko et al. (2014) dans les forêts de montagnes du Parc National de Kahuzi-Biega en République Démocratique du Congo où son abondance est plus élevée dans les bas-fonds qu'aux sommets (effet négatif de l'altitude). Par ailleurs, les résultats de la présente étude corroborent ceux de Parthasarathy et al. (2004) qui ont mis en exergue la diminution de l'abondance des lianes avec l'augmentation de l'altitude dans les forêts tropicales humides de la Péninsule indienne. L'effet positif de la superficie de la trouée sur l'abondance des lianes a été également mis en évidence par Babweteera et al. (2000) qui ont montré que la densité des lianes augmente avec l'augmentation de la surface des trouées dans la Réserve Forestière de Budongo en Uganda. Il est indiqué que l'âge des trouées est positivement corrélé à l'abondance et la diversité des lianes (Babweteera et al., 2000). Cet aspect n'ayant pas été abordé dans le cadre de cette étude, il est suggéré une étude qui tiendrait compte de ce facteur pour élucider sa relation avec l'abondance de $S$. scandens.

Les résultats ont également renseigné sur un effet positif de la pente sur l'abondance de $S$. scandens. Comme précédemment annoncé, la liane est plus abondante dans les zones de faible altitude. Or, ces dernières sont essentiellement rencontrées au côté du bassin versant du Congo où des valeurs d'altitude allant de 1600 à 1900 m ont été observées avec des pentes davantage marquées (Habonayo et al., 2019). Cette situation expliquerait l'effet positif constaté de la pente sur l'abondance de la liane.

\section{Envahissement de Sericostachys scandens et diversité des herbacées}

Les résultats ont montré que la densité de $S$. scandens influence négativement la richesse spécifique, la diversité en genres et la diversité en familles des herbacées du PNK. Ces résultats sont en accord avec ceux de Liengola (2008) ayant indiqué que l'envahissement de $S$. scandens a un effet négatif sur la richesse spécifique des herbacées et arbustes/arbres dans les forêts de montagnes du Parc National de Kahuzi-Biega en République Démocratique du Congo. Les plantes concurrencent leurs voisins pour un ensemble fini de ressources rares; ce qui diminue la performance, la croissance et la survie des plantes individuelles (Wright et al., 2015). S. scandens rivaliserait intensément avec la végétation en association avec elle et ainsi limiterait le nombre potentiel d'espèces $\mathrm{du}$ milieu où elle est en plein essor.

Les résultats ont également montré une variation significative de la composition floristique des herbacées entre les trouées envahies par la liane et les parcelles témoins. Cette situation corrobore le constat de Liengola (2008) qui a révélé que l'invasion par $S$. scandens entraîne une variation de la composition floristique dans les forêts de montagnes du parc ci-haut indiqué.

Enfin, les résultats ont mis en relief que les espèces les plus indicatrices (valeur indicatrice $\geq 0,80$ ) des trouées envahies par $S$. scandens sont des espèces pionnières (héliophiles) caractéristiques des milieux 
perturbés ou des premiers stades de reconstitution forestière. Pteridium aquilinum a été reconnue comme indicateur biologique important des sites dégradés du Parc National de la Kibira (Habonayo et Ndihokubwayo, 2012) et comme espèce post-culturale des jachères du Burundi (Bangirinama, 2010). Ce dernier auteur a classé également Ageratum conyzoides et Rubus apetalus dans les espèces post-culturales. Les espèces telles que Gynura scandens, Urera hypselodendron, Clerodendrum johnstonii, Crassocephalum montuosum, Triumfetta tomentosa et Ipomoea involucrata ont été rapportées comme caractéristiques de reconstitution forestière du Parc National de la Kibira au stade pionnier et de friches herbacées (Habiyaremye et Nzigidahera, 2016). Balezi et al. (2008) ont aussi signalé que la prolifération de $S$. scandens entraîne l'expansion des espèces des milieux perturbés.

$\mathrm{Au}$ contraire, les espèces indicatrices des parcelles non envahies par la liane sont des espèces d'ombre caractéristiques des milieux non perturbés ou des stades plus avancées de reconstitution forestière. En effet, Habiyaremye et Nzigidahera (2016) ont rapporté que Brillantaisia cicatricosa, Chassalia subochreata, Asplenium elliottii, Calanthe sylvatica, Pilea rivularis, Elatostema monticola, Galiniera coffeoides, Impatiens burtonii, Notholaena inaequalis et Rutidea orientalis, Piper capense, Achyrospermum micranthum et Sanicula elata, sont des espèces sciaphiles typiques des vieilles forêts secondaires et/ou des forêts primaires où la luminosité au sol est faible. Ainsi donc, en considérant à la fois l'action de l'homme impliqué dans l'expansion de $S$. scandens (Masumbuko, 2011) et l'action transformatrice de cette liane sur les communautés des herbacées, il y a lieu de retenir que $S$. scandens est une conséquence et non une cause de la perturbation des communautés des herbacées, mais aussi qu'elle aggrave les effets des perturbations anthropogènes et les rend sans doute plus durables dans le temps.

\section{Conclusion}

Sericostachys scandens est en plein développement dans les trouées du PNK où son abondance est essentiellement influencée par la superficie des trouées et l'altitude. La densité de la liane augmente avec la taille des trouées, mais diminue au fur et à mesure que l'on monte en altitude. L'invasion par S. scandens entraîne une variation de la composition floristique entre les trouées et les zones non envahies sous canopée intacte, ces dernières étant caractérisées par une diversité élevée comparativement aux trouées. En outre, l'invasion par S. scandens s'accompagne d'une prolifération des espèces héliophiles dans les trouées, lesquelles sont caractéristiques de stations perturbées. Le PNK devrait faire objet d'une gestion plus rigoureuse pour barrer la route aux actions perturbatrices en particulier celles qui conduisent à la création des trouées.

\section{CONFLIT D'INTERETS}

Les auteurs de cet article déclarent qu'il n'y a aucun conflit d'intérêts dans cette publication.

\section{CONTRIBUTIONS DES AUTEURS}

Ce manuscrit porte sur un des aspects d'une thèse en cours de réalisation par RH sous l'encadrement de $\mathrm{ACA}$ et $\mathrm{BH}$, respectivement directeur et co-directeur de thèse. Les autres auteurs du manuscrit sont AFA (un des deux co-premiers auteurs pour sa contribution dans le traitement statistique des données et la lecture du manuscrit), GHD (pour l'avoir lu en vue de l'amélioration de sa qualité) et FH (pour avoir contribué dans l'amélioration du protocole de recherche doctorale, la détermination des noms scientifiques des espèces et la lecture du manuscrit).

\section{REMERCIEMENTS}

Les auteurs expriment leur gratitude à l'endroit de la Direction Générale de l'Office Burundais pour la Protection de l'Environnement (OBPE) qui a délivré l'autorisation de mener cette étude dans le Parc National de la Kibira. Une profonde reconnaissance est également témoignée aux 
gardes forestiers dudit Parc National pour leur aide sur le terrain.

\section{REFERENCES}

Asner GP, Martin RE. 2012. Contrasting leaf chemical traits in tropical lianas and trees: implications for future forest composition. Ecol. Lett., 15 (9): 10011007. DOI: $10.1111 / \mathrm{j} .1461-$ 0248.2012.01821.x

Babweteera F, Plumptre A, Obua J. 2000. Effect of gap size and age on climber abundance and diversity in Budongo Forest Reserve, Uganda. Afr. J. Ecol., 38 (3): 230-237. DOI: https://doi.org/10.1046/j.13652028.2000.00245.x

Balezi Z, Nyakabwa M, Lejoly J. 2008. Etude écologique de la liane envahissante Sericostachys scandens dans la partie de haute altitude du Parc National de Kahuzi-Biega (PNKB), (Sud-Kivu, R.D.Congo). Annales de la Faculté des Sciences de l'Université Officielle de Bukavu, 1 (1): 28-36.

Bangirinama F. 2010. Processus de la restauration écosystémique au cours de la dynamique post-culturale au Burundi: mécanisme, caractérisation et séries écologiques. Thèse de doctorat, ULB, Bruxelles, p. 185.

Bongers F, Ewango CEN. 2015. Dynamics of lianas in D R Congo. In Ecology of Lianas, Schnitzer SA, Bongers F, Burnham RJ, Putz FE (eds). WileyBlackwell Publishing: Oxford; 23-35.

Cazenave-Piarrot F, Cazenave-Piarrot A, Albert L. 1979. Géographie du Burundi. Le pays et les Hommes. Edicef: Paris.

Dhetchuvi MJB, Gakwavu RJ. 2006. Etude préliminaire de la régénération en forêt de montagne du Rwanda: cas de huit espèces d'arbres illégalement exploitées en Forêt de Nyungwe. Actes de la Deuxième Conférence Annuelle de la Recherche Scientifique, Université Nationale du Rwanda, 7-21.

Dufrêne M, Legendre P. 1997. Species assemblages and indicator species definition: the need of an asymmetrical and flexible approach. Ecol. Monogr., 67 (3): 345-366. DOI: 10.2307/2963459

Gerwing JJ, Schnitzer SA, Burnham RJ, Bongers F, Chave J, DeWalt SJ, Ewango CEN, Foster R, Kenfack D, MartinezRamos M, Parren M, Parthasarathy N, Pérez-Salicrup DR, Putz FE, Thomas DW. 2006. A standard protocol for liana censuses. Biotropica, 38 (2): 256-261. DOI: 10.1111/j.1744-7429.2006.00134.x Gourlet S. 1986. Le Parc National de la Kibira. Quelle Potentialité pour quel Avenir? ENGREF: Paris.

Grainger JM, van Aarde RJ. 2012. The role of canopy gaps in the regeneration of coastal dune forest. Afr. J. Ecol., 51: 11-20. DOI: https://doi.org/10.1111/j.13652028.2012.01348.x

Habiyaremye MF, Nzigidahera B. 2016. Habitat du Parc National de la Kibira (Burundi). Lexique des Plantes pour Connaître et Suivre l'Évolution des Forêts du Secteur Rwegura. Institut Royal des Sciences Naturelles de Belgique: Bruxelles.

Habonayo R, Ndihokubwayo R. 2012. Détermination d'indicateurs de dégradation du Parc National de la Kibira (Burundi): cas du secteur Rwegura. Mémoire de Master, Université du Burundi, Bujumbura, p. 92 p.

Habonayo R, Azihou AF, Dassou GH, Adomou AC, Habonimana B. 2019 Facteurs déterminant l'abondance de Sericostachys scandens (Amaranthaceae) dans le Parc National de la Kibira au Burundi. J. Anim. Plant. Sci., 39 (1): 6359-6371.

IGEBU (Institut Géographique du Burundi). 2001. Bulletin climatologique mensuel (1990-2001). Burundi, Gitega.

IGEBU (Institut Géographique du Burundi). 2015. Bulletin climatologique mensuel (1986-2015). Burundi, Gitega.

INECN (Institut National pour l'Environnement et la Conservation de la Nature). 1992. Plan de Gestion du Parc National de la Kibira. INECN: Gitega.

Krug O. 1993. Etude des Systèmes de Production et des Systèmes Agraires des 
trois Communes Riveraines $d u$ PNK: Proposition en vue d'une Réduction des Conflits. INECN: Gitega.

Laurance WF, Pérez D, Delamônica P, Fearnside PM, Agra S, Jerozolinski A, Pohl L, Lovejoy TE. 2001. Rain forest fragmentation and the structure of amazonian liana communities. Ecology, 82 (1): 105-116. DOI: $10.2307 / 2680089$

Ledo A, Schnitzer SA. 2014. Disturbance and clonal reproduction determine liana distribution and maintain liana diversity in a tropical forest. Ecology, 95 (8): 2169 2178. DOI: $10.1890 / 13-1775.1$.

Lewalle J. 1972. Les étages de la végétation du Burundi Occidentale. Bull. Jard. Bot. Nat. Belg., 42: 1-247.

Liengola I. 2008. Influence of the invasive liana Sericostachys scandens on forest composition: Implications for the recovery of Grauer's Gorilla in the Kahuzi-Biega National Park, Democratic Republic of Congo. Trop. Resour. Bull., 27: 43-50.

Masumbuko NC. 2011. Ecologie de Sericostachys scandens, liane envahissante dans les forêts de montagne du Parc National de Kahuzi-Biega, République Démocratique du Congo. Thèse de doctorat, ULB, Bruxelles, $\mathrm{p}$. 192.

Masumbuko NC, Habiyaremye M, Meerts P. 2014. Density of lianas following the topo-sequence in the Mountainous Rainforest of Kahuzi-Biega National Parc. In Mountains, Gonçalves AJB, Vieira AAB (eds). Nova Science Publishers: New York; 251-262.

Meddour R. 2011. La Méthode Phytosociologique Sigmatiste ou BraunBlanqueto-Tüxenienne. Université Mouloud Mammeri: Tizi Ouzou.

MEEATU (Ministère de l'Eau, de l'Environnement, de l'Aménagement du Territoire et de l'Urbanisme) 2013. Plan régional de mise en œuvre de la Stratégie Nationale et Plan d'Action sur la Biodiversité en zones écologiques de
Mumirwa et de la Crête Congo-Nil. MEEATU: Bujumbura.

Nakazawa M. 2017fmsb. Functions for Medical Statistics Book with some Demographic Data. R package version 0.6.1. https://CRAN.Rproject.org/package $=$ fmsb

Oksanen J, Blanchet FG, Friendly M, Kindt R, Legendre P, McGlinn D, Minchin PR, O'Hara RB, Simpson GL, Solymos P, Stevens MHH, Szoecs E, Wagner H. 2017vegan. Community Ecology Package. $R$ package version 2.4-4. https://CRAN.R-

project.org/package=vegan

Parthasarathy N, Muthuramkumar S, Sridhar Reddy M. 2004. Patterns of liana diversity in tropical evergreen forests of peninsular India. For. Ecol. Manage., 190: $15-31$. DOI: 10.1016/j.foreco.2003.10.003

R Core Team. 2017 R. A language and environment for statistical computing. $\mathrm{R}$ Foundation for Statistical Computing, Vienna, Austria. URL https://www.Rproject.org/.

Reekmans M, Niyongere L. 1983. Lexique Vernaculaire des Plantes Vasculaires $d u$ Burundi. Travaux de la Faculté des Sciences. Université du Burundi: Bujumbura.

Roberts DW. 2016 labdsv. Ordination and Multivariate Analysis for Ecology. R package version 1.8-0. https://CRAN.Rproject.org/package $=$ labdsv

Schnitzer SA, Dalling JW, Carson WP. 2000. The impact of lianas on tree regeneration in tropical forest canopy gaps: Evidence for an alternative pathway of gap-phase regeneration. J. Ecol., 88 (4): 655-666. DOI: $10.1046 / \mathrm{j} .1365-2745.2000 .00489 . x$

Schnitzer SA, Carson WP. 2010. Lianas suppress tree regeneration and diversity in treefall gaps. Ecol. Lett., 13: 849-857. DOI: 10.1111/j.1461-0248.2010.01480.x

Schnitzer SA, van der Heijden G, Mascaro J, Carson WP. 2014. Lianas in gaps reduce carbon accumulation in a tropical forest. 
Ecology, 95 (11): 3008-3017. DOI: https://doi.org/10.1890/13-1718.1

Schnitzer SA, Putz FE, Bongers F, Kroening K. 2015. The past, present, and potential future of liana ecology. In Ecology of Lianas, Schnitzer SA, Bongers F, Burnham RJ, Putz FE (eds). WileyBlackwell Publishing: Oxford; 3-12.

Senterre B. 2005. Recherches méthodologiques pour la typologie de la végétation et la phytogéographie des forêts denses d'Afrique tropicale. Thèse de doctorat, ULB, Bruxelles, p. 345.

Yorke, SR, Schnitzer SA, Mascaro J, Letcher S, Carson WP. 2013. Increasing liana abundance and basal area in a tropical forest: the contribution of long distance clonal colonization. Biotropica, 45 (3): 317-324.

DOI: https://doi.org/10.1111/btp.12015
Van der Heijden GMF, Schnitzer SA, Powers JS, Phillips OL. 2013. Liana impacts on carbon cycling, storage and sequestration in tropical forests. Biotropica, 45 (6): 682-692.

DOI: https://doi.org/10.1111/btp.12060

Venables WN, Ripley BD. 2002. Modern Applied Statistics with $S$ (Fourth Edition). Springer, New York. ISBN 0-387-954570

Wright A, Schnitzer SA, Reich PB. 2015. Daily environmental conditions determine the competition-facilitation balance for plant water status. J. Ecol., 103: 648-656. DOI: 10.1111/1365-2745.12397

Wyka TP, Oleksyn J, Karolewsk P, Schnitzer SA. 2013. Phenotypic correlates of the lianescent growth form: a review. Ann. Bot., 112: 1667-1681. 